\title{
Research on Fracture Toughness of C120 Ultra-High-Performance Concrete in Kingkey Financial Center Project
}

\author{
Hao-Wen Ye, ${ }^{1}$ Nai-Qian Feng, ${ }^{2}$ Zhi-Wei Ran, ${ }^{1}$ Li-Xun Lin, ${ }^{1}$ Yan Ling-Hu, ${ }^{1}$ \\ Shi-Kun Qi, ${ }^{1}$ and Yi Dong ${ }^{1}$ \\ ${ }^{1}$ Technology Department, China Construction Fourth Engineering Division Corp. Ltd., Guangzhou 510665, China \\ ${ }^{2}$ Senior Scientists Association, Tsinghua University, Beijing 100084, China \\ Correspondence should be addressed to Yan Ling-Hu, linghuyan9@hotmail.com
}

Received 13 December 2011; Accepted 18 February 2012

Academic Editor: Jin Ping Lu

Copyright ( 2012 Hao-Wen Ye et al. This is an open access article distributed under the Creative Commons Attribution License, which permits unrestricted use, distribution, and reproduction in any medium, provided the original work is properly cited.

By adding polypropylene fiber, fracture toughness of C120 ultra-high-performance concrete in Kingkey Financial Center project has been enhanced. The tests conducted by the Building Material Lab of the Civil and Water Conservancy Institute of Tsinghua University provided satisfactory results of mechanical property and fracture toughness of C120 ultra-high-performance concrete.

\section{Preface}

In order to understand the mechanical property of C120 ultra-high-performance concrete under static modulus of elasticity and anticracking properties, including fracture toughness, the research group of C120 ultra-highperformance concrete conducted a series of tests in the Building Material Lab of the Civil Water Conservancy Institute of Tsinghua University.

\section{Experimental Details}

2.1. Raw Materials. Following raw materials are used in this research: (1) cement (Nanjing Xiaoyetian PII52.5), (2) microballoon (Made in Kunming), (3) ganister sand (from Zhunyi, Guizhou), (4) Fine aggregate (sea sand, desalted, FM2.6 2.8), (5) coarse aggregate (macadam, 5$10,10-20 \mathrm{~mm}$ in diameter), (6) Water reducer (BASF poly carboxylic acid, solid content: 40\%, made in Guangdong), (7) water (Shenzhen tap water), and (8) polypropylene fiber (Grace $19 \mathrm{~mm}$ long fiber and short fiber provided by Shenzhen Lijian Concrete Company).

2.2. Mix Proportions. Please refer to Figure 1. This test aims to see what the difference is in concrete with long fibers and short fibers, so the compositions of every group of test pieces are the same, except fiber types and quantities.
2.3. Mixing Process. Get certain amount of raw materials ready. At first, put gelatinizer and sand into a mixer and run for 1 minute; then add water and additive, mix for 2 minutes; lastly, put in coarse aggregate, mix for 1 minute. For materials that are too sticky, mix 1 more minute after adding coarse aggregate. Find Mix Proportions in Table 1.

2.4. Specimens. Below specimens are used in the research:

$$
\begin{aligned}
& 100 \mathrm{~mm} \times 100 \mathrm{~mm} \times 400 \mathrm{~mm} \text { prisms (3), } \\
& 100 \mathrm{~mm} \times 100 \mathrm{~mm} \times 300 \mathrm{~mm} \text { prisms (6), } \\
& 100 \mathrm{~mm} \times 100 \mathrm{~mm} \times 100 \mathrm{~mm} \text { cubes (3). }
\end{aligned}
$$

2.5. Curing. Upon being demoulded, specimens were transferred to the caring unit and soaked into calcarea hydrica solution (lime water) for 28 days. Finished specimens were immediately sent to the Building Material Lab of the Civil Water Conservancy Institute of Tsinghua University.

\section{Main Mechanical Property Tests}

Results of cube compression test, prismoid compression test, and static modulus of elasticity test have been listed in Figures 1 and 2. Equipment used in the tests included YE200A hydraulic pressure test machine, YJR-5 static digital 
TABLE 1: Mix proportions.

\begin{tabular}{|c|c|c|c|c|c|c|c|c|}
\hline No. & $\mathrm{W} / \mathrm{B}$ & $\mathrm{C}$ & $\mathrm{SF}+\mathrm{FA}+\mathrm{BFS}$ & $S$ & G1 & G2 & Polycarboxylic acid additive & Fibers \\
\hline 1 & 0.17 & 550 & 150 & 750 & 285 & 665 & $2.5 \%$ & No fiber \\
\hline 2 & 0.17 & 550 & 150 & 750 & 285 & 665 & $2.5 \%$ & Long fiber, $1 \mathrm{~kg} / \mathrm{m}^{3}$ \\
\hline 3 & 0.17 & 550 & 150 & 750 & 285 & 665 & $2.5 \%$ & Long fiber, $2 \mathrm{~kg} / \mathrm{m}^{3}$ \\
\hline 4 & 0.17 & 550 & 150 & 750 & 285 & 665 & $2.5 \%$ & Short fiber, $2 \mathrm{~kg} / \mathrm{m}^{3}$ \\
\hline
\end{tabular}

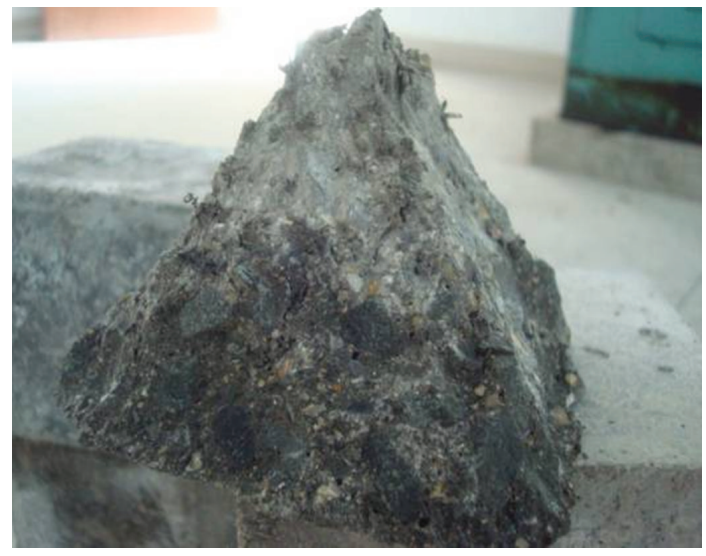

Figure 1: Damaged C120 concrete.

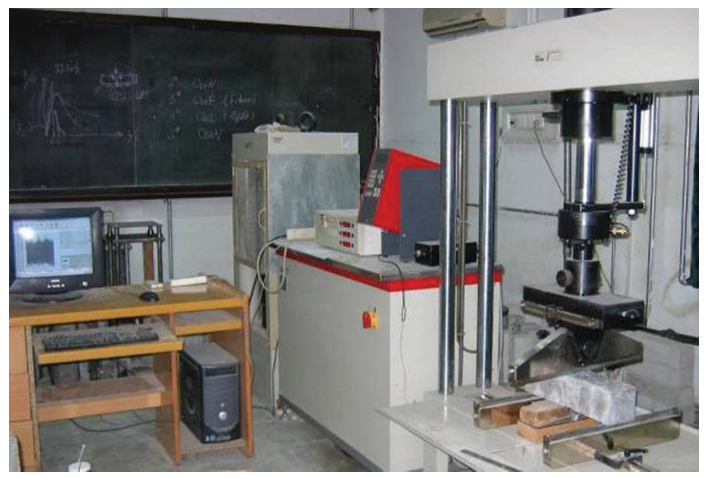

FIGURE 2: C120 high-performance concrete fracture parameter test.

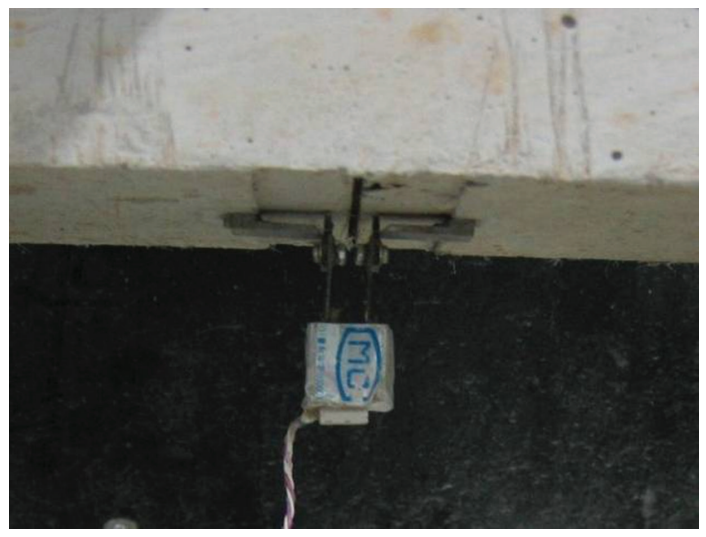

Figure 3: Crack opening displacement test.

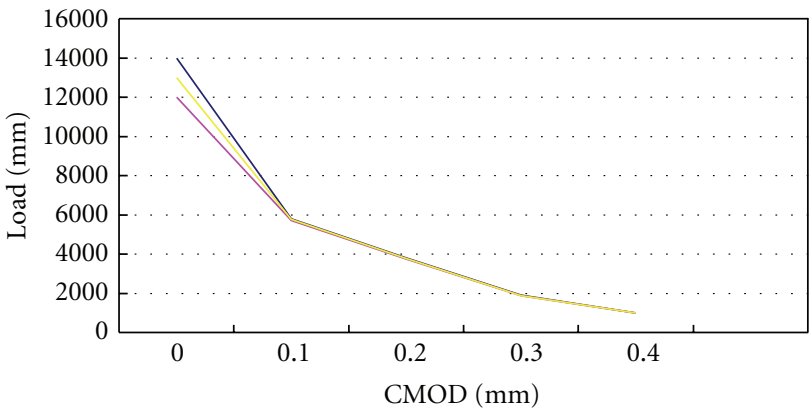

- Norm 1

- Norm 2

- Norm 3

Figure 4: P-CMOD for basic concrete.

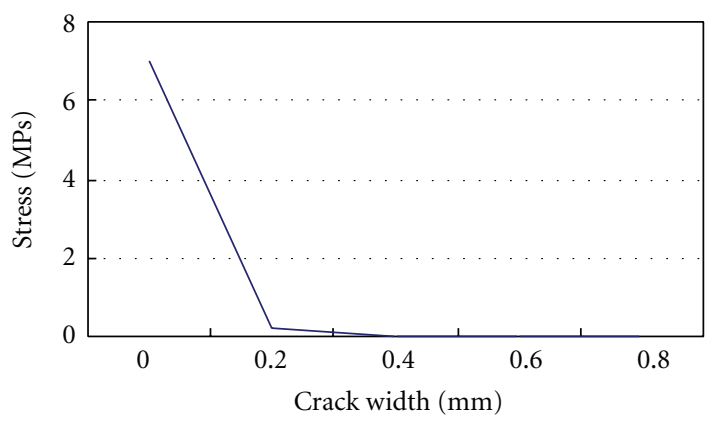

— Norm 1

Figure 5: $\sigma$-w for basic concrete.

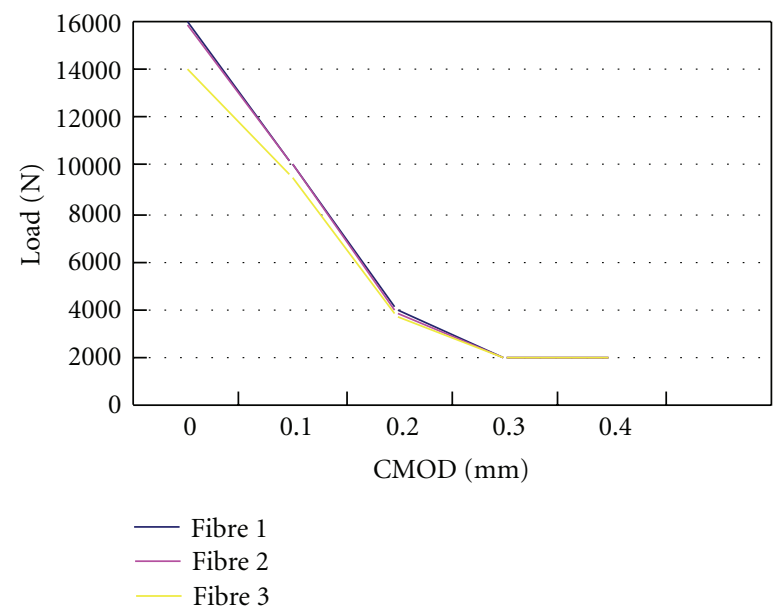

FIgURE 6: P-CMOD for concrete with $1 \mathrm{~kg}$ long fiber. 


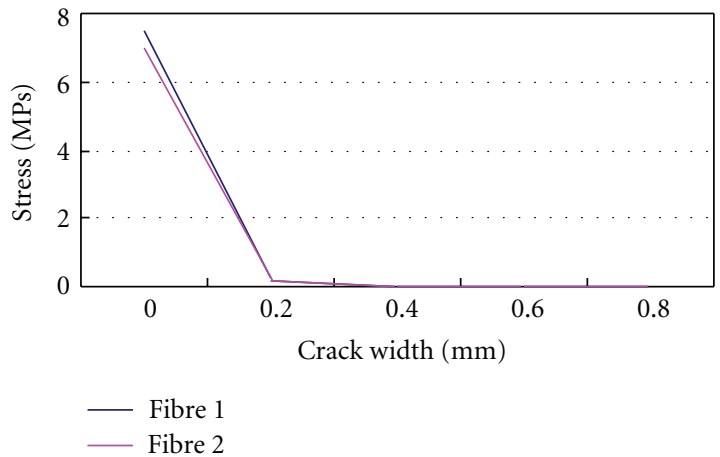

FIGURE 7: $\sigma$-w for concrete with $1 \mathrm{~kg}$ long fiber.

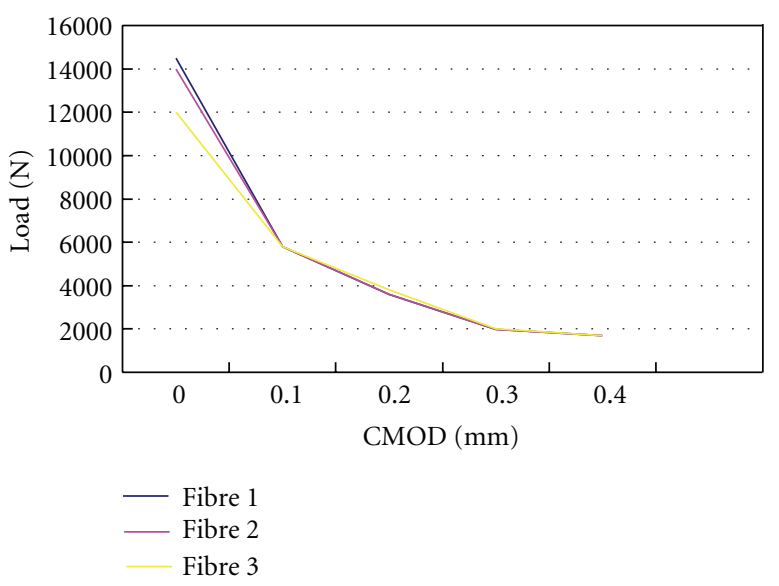

FIgURE 8: P-CMOD for concrete with $2 \mathrm{~kg}$ long fiber.

strain gauging unit, and YEW-200A dropping electrode compression-testing machine (Tables 2 and 3).

\section{C120 Concrete Fracture Toughness Test}

4.1. Equipment. Toni2071 Pressure Flexure Compression Testing Machine was made in Germany, Maximum Capacity $200 \mathrm{KN}$, for flexure and crack opening displacement tests under constant rate of loading or rate of displacement.

\subsection{C120 Concrete Fracture Parameter Test. See Figures 2 and} 3.

4.3. P-CMOD and $\sigma-w$ Curve Charts for 4 Types of Concrete. Through tests, we got the P-CMOD (loading-crack opening displacement) curve charts for basic concrete, concrete with $1 \mathrm{~kg}$ long fiber, concrete with $2 \mathrm{~kg}$ long fiber, and concrete with $2 \mathrm{~kg}$ short fiber, on which we came up with the O-W (softening relation) curve charts (Table 4).

\section{Analysis}

(1) From Figures 4, 5, 6, 7, 8, 9, 10, 11, 12, 13, 14, and 15 , we could tell that P-CMOD and O-W curves were similar for the same type of concrete, which meant test piece's dispersion was not large.

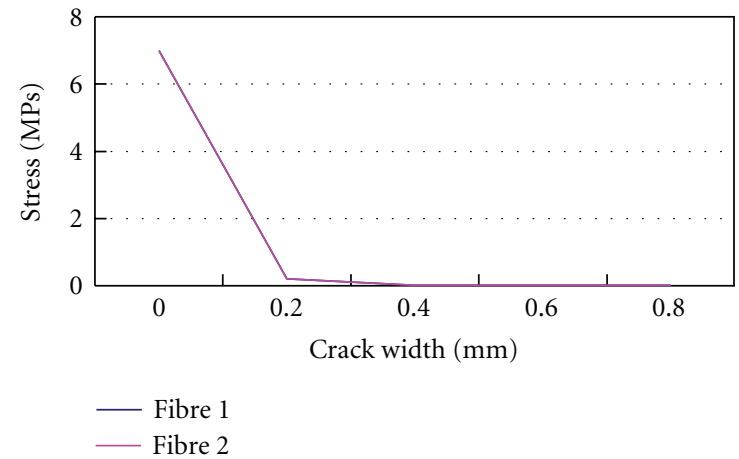

FIgURE 9: $\sigma$-w for concrete with $2 \mathrm{~kg}$ long fiber.

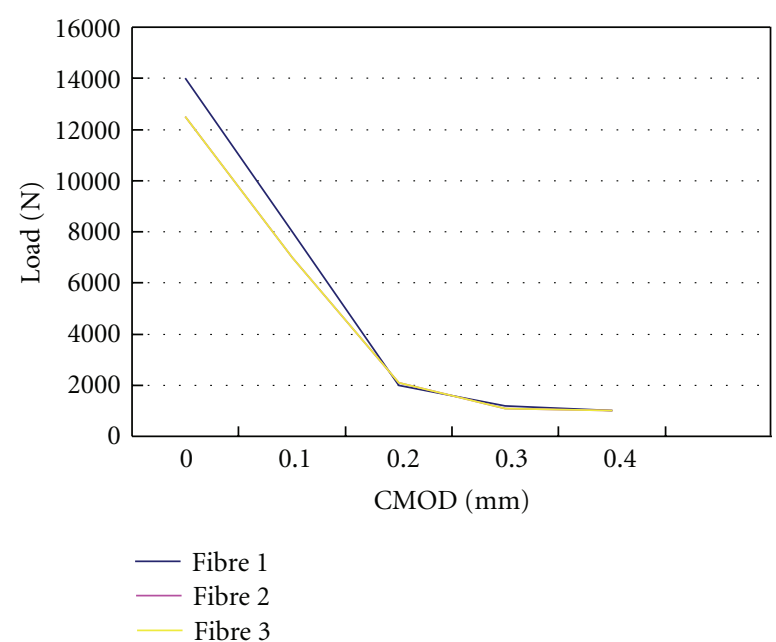

Figure 10: P-CMOD for concrete with $2 \mathrm{~kg}$ short fiber.

(2) 4 types of concrete were almost the same, except fiber content, which only presented difference after cracking. Therefore, the cracking load for all 4 types of concrete was similar.

(3) As fiber content increased, concrete's tensile strength almost remained the same.

(4) As fiber content increased, concrete's cracking energy mounted too. When the fiber content remained the same, fiber type played a role in cracking difference. Test results suggested that cracking energy rose in order of basic concrete < concrete with $1 \mathrm{~kg}$ long fiber $<$ concrete with $2 \mathrm{~kg}$ long fiber < concrete with $2 \mathrm{~kg}$ short fiber.

(5) Characteristic length of brittleness " $l_{\mathrm{ch}}$ " notates the brittleness of concrete. The smaller the number is, the more brittle the concrete is. From the test results, we learnt that as fiber content increased, concrete's toughness enhanced too. Characteristic length of brittleness varies in order: basic concrete $<$ concrete with $1 \mathrm{~kg}$ long fiber < concrete with $2 \mathrm{~kg}$ short fiber $<$ concrete with $2 \mathrm{~kg}$ long fiber, and toughness varies in order:basic concrete < concrete with $1 \mathrm{~kg}$ long fiber 
TABLE 2: Compressive strength test results of cube specimens.

\begin{tabular}{|c|c|c|c|c|}
\hline No. & Age & Size & Individual value & Compression strength \\
\hline \multirow{3}{*}{ 1: Initial } & \multirow{3}{*}{28 days } & \multirow{3}{*}{$100 \times 100 \times 100 \mathrm{~mm}$} & 127.0 MPa & \multirow{3}{*}{ 124.0 MPa } \\
\hline & & & $121.0 \mathrm{MPa}$ & \\
\hline & & & $124.0 \mathrm{MPa}$ & \\
\hline \multirow{3}{*}{ 2: $1 \mathrm{~kg}$ long fiber } & \multirow{3}{*}{28 days } & \multirow{3}{*}{$100 \times 100 \times 100 \mathrm{~mm}$} & $129.0 \mathrm{MPa}$ & \multirow{3}{*}{$124.7 \mathrm{MPa}$} \\
\hline & & & 126.0 MPa & \\
\hline & & & $119.0 \mathrm{MPa}$ & \\
\hline \multirow{3}{*}{ 3: $2 \mathrm{~kg}$ long fiber } & \multirow{3}{*}{28 days } & \multirow{3}{*}{$100 \times 100 \times 100 \mathrm{~mm}$} & $140.0 \mathrm{MPa}$ & \multirow{3}{*}{$135.3 \mathrm{MPa}$} \\
\hline & & & $123.0 \mathrm{MPa}$ & \\
\hline & & & $141.0 \mathrm{MPa}$ & \\
\hline \multirow{2}{*}{ 4: $2 \mathrm{~kg}$ short fiber } & \multirow{2}{*}{28 days } & \multirow{2}{*}{$100 \times 100 \times 100 \mathrm{~mm}$} & $120.0 \mathrm{MPa}$ & \multirow{2}{*}{$118.0 \mathrm{MPa}$} \\
\hline & & & $115.0 \mathrm{MPa}$ & \\
\hline
\end{tabular}

TABLE 3: Results of compressive strength and static modulus of elasticity of prism specimens.

\begin{tabular}{|c|c|c|c|c|c|c|}
\hline \multirow{2}{*}{ No. } & \multirow{2}{*}{ Age } & \multirow{2}{*}{ Size } & \multicolumn{2}{|c|}{ Compression strength } & \multicolumn{2}{|c|}{ Static modulus of elasticity } \\
\hline & & & Individual & Average & Individual & Average \\
\hline \multirow{3}{*}{ 1: Initial } & \multirow{3}{*}{ 28天 } & \multirow{3}{*}{$100 \times 100 \times 300$} & $115.2 \mathrm{MPa}$ & \multirow{3}{*}{ 138.0 MPa } & $5.20 \times 10^{4} \mathrm{MPa}$ & \multirow{3}{*}{$5.20 \times 10^{4} \mathrm{MPa}$} \\
\hline & & & $138.0 \mathrm{MPa}$ & & $5.18 \times 10^{4} \mathrm{MPa}$ & \\
\hline & & & $138.8 \mathrm{MPa}$ & & $5.22 \times 10^{4} \mathrm{MPa}$ & \\
\hline \multirow{3}{*}{ 2: $1 \mathrm{~kg}$ long fiber } & \multirow{3}{*}{ 28天 } & \multirow{3}{*}{$100 \times 100 \times 300$} & $127.2 \mathrm{MPa}$ & \multirow{3}{*}{$128.7 \mathrm{MPa}$} & $5.10 \times 10^{4} \mathrm{MPa}$ & \multirow{3}{*}{$5.23 \times 10^{4} \mathrm{MPa}$} \\
\hline & & & $126.8 \mathrm{MPa}$ & & $5.21 \times 10^{4} \mathrm{MPa}$ & \\
\hline & & & $132.0 \mathrm{MPa}$ & & $5.37 \times 10^{4} \mathrm{MPa}$ & \\
\hline \multirow{3}{*}{$3: 2 \mathrm{~kg}$ long fiber } & \multirow{3}{*}{ 28天 } & \multirow{3}{*}{$100 \times 100 \times 300$} & $137.2 \mathrm{MPa}$ & \multirow{3}{*}{$136.6 \mathrm{MPa}$} & $5.22 \times 10^{4} \mathrm{MPa}$ & \multirow{3}{*}{$5.30 \times 10^{4} \mathrm{MPa}$} \\
\hline & & & $139.0 \mathrm{MPa}$ & & $5.22 \times 10^{4} \mathrm{MPa}$ & \\
\hline & & & $133.6 \mathrm{MPa}$ & & $5.45 \times 10^{4} \mathrm{MPa}$ & \\
\hline \multirow{3}{*}{ 4: $2 \mathrm{~kg}$ short fiber } & \multirow{3}{*}{ 28天 } & \multirow{3}{*}{$100 \times 100 \times 300$} & $104.8 \mathrm{MPa}$ & \multirow{3}{*}{ 116.7 $\mathrm{MPa}$} & $5.18 \times 10^{4} \mathrm{MPa}$ & \multirow{3}{*}{$5.25 \times 10^{4} \mathrm{MPa}$} \\
\hline & & & $116.4 \mathrm{MPa}$ & & $5.23 \times 10^{4} \mathrm{MPa}$ & \\
\hline & & & $128.8 \mathrm{MPa}$ & & $5.33 \times 10^{4} \mathrm{MPa}$ & \\
\hline
\end{tabular}

TABLE 4: Concrete fracture parameter test results.

\begin{tabular}{|c|c|c|c|c|c|c|c|c|c|c|}
\hline Type & No. & $E(\mathrm{GPa})$ & $P_{f c}(N)$ & $P_{\max }(N)$ & $\sigma_{c}(\mathrm{MPa})$ & $\sigma_{f c}(\mathrm{MPa})$ & $\sigma_{t}(\mathrm{MPa})$ & $\sigma_{f}(\mathrm{MPa})$ & $G_{f}\left(\mathrm{~J} / \mathrm{m}^{2}\right)$ & $l_{\mathrm{ch}}(\mathrm{cm})$ \\
\hline \multirow{4}{*}{ Basic } & 1 & \multirow{4}{*}{51.9} & 5000 & 14000 & 127.0 & 6.82 & 6.86 & 9.07 & 213.8 & 23.5 \\
\hline & 2 & & 4600 & 14500 & 121.0 & 6.27 & - & 9.39 & - & - \\
\hline & 3 & & 4700 & 13800 & 124.0 & 6.41 & - & 9.59 & - & - \\
\hline & Avg. & & 4767 & 14100 & 124.0 & 6.50 & 6.86 & 9.35 & 213.8 & 23.5 \\
\hline \multirow{4}{*}{$1 \mathrm{~kg}$ long fiber } & 1 & \multirow{4}{*}{52.2} & 4800 & 13800 & 129.0 & 6.55 & 6.67 & 8.94 & 291.8 & 33.8 \\
\hline & 2 & & 5000 & 15500 & 126.0 & 6.82 & 7.08 & 10.04 & 302.9 & 27.9 \\
\hline & 3 & & 5000 & 15300 & 119.2 & 6.82 & - & 9.91 & - & - \\
\hline & Avg. & & 4933 & 14867 & 124.7 & 6.73 & 6.88 & 9.63 & 297.35 & 30.9 \\
\hline \multirow{4}{*}{$2 \mathrm{~kg}$ long fiber } & 1 & \multirow{4}{*}{52.2} & 4800 & 12000 & 140.8 & 6.55 & 6.56 & 7.78 & 322.8 & 39.2 \\
\hline & 2 & & 5000 & 15000 & 123.6 & 6.82 & - & 9.73 & - & - \\
\hline & 3 & & 5200 & 14000 & 141.4 & 7.09 & 7.22 & 9.08 & 322.1 & 33.4 \\
\hline & Avg. & & 5000 & 13667 & 135.3 & 6.82 & 6.89 & 8.86 & 322.5 & 36.3 \\
\hline \multirow{4}{*}{$2 \mathrm{Kg}$ short fiber } & 1 & \multirow{4}{*}{52.0} & 5100 & 14000 & 120.0 & 6.95 & 6.97 & 9.07 & 300.3 & 30.0 \\
\hline & 2 & & 5000 & 14000 & 115.0 & 6.82 & 6.84 & 9.07 & 321.8 & 30.7 \\
\hline & 3 & & 5200 & 14000 & - & 7.09 & - & 9.07 & - & - \\
\hline & Avg. & & 5100 & 14000 & 118.0 & 6.95 & 6.91 & 9.07 & 311.1 & 30.4 \\
\hline
\end{tabular}

$\left(P_{f_{c}}=\right.$ cracking load, $P_{\max }=$ maximum load, $E=$ modulus of elasticity, $\sigma_{c}=$ compression strength, $\sigma_{f c}=$ cracking strength, $\sigma_{t}=$ tensile strength, $\sigma_{f}=$ flexural strength, $G_{f}=$ fracture energy, $l_{\mathrm{ch}}=$ brittleness; the smaller the $l_{\mathrm{ch}}$ is, the more brittle the concrete is) 


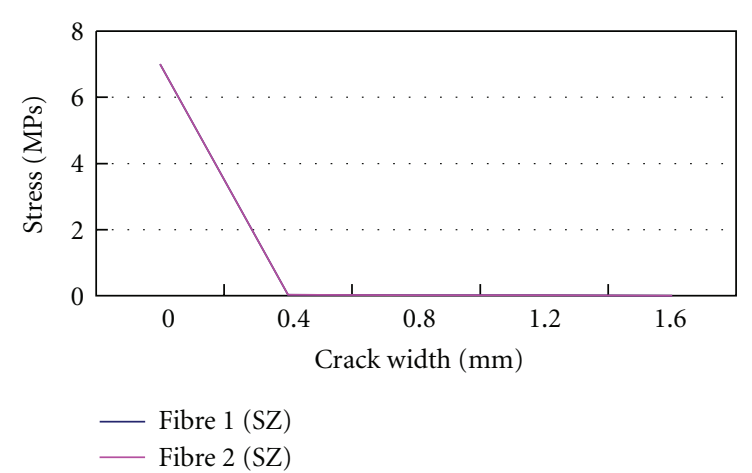

FIGURE 11: $\sigma$-w for concrete with $2 \mathrm{~kg}$ short fiber.

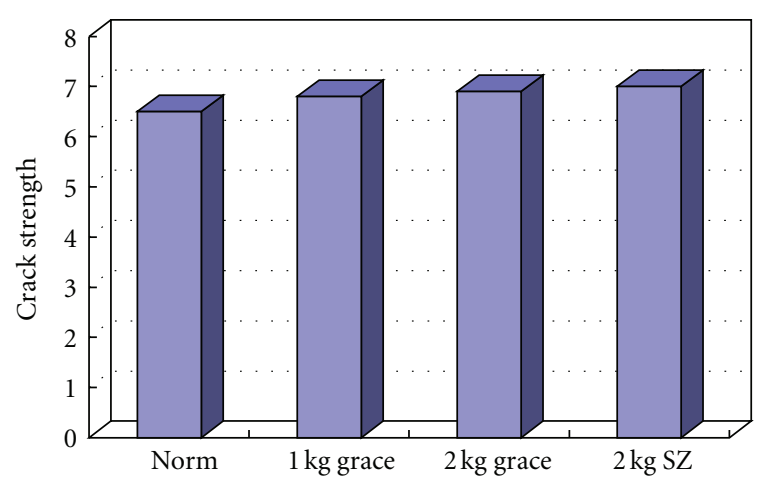

FIGURE 12: Cracking strength comparison.

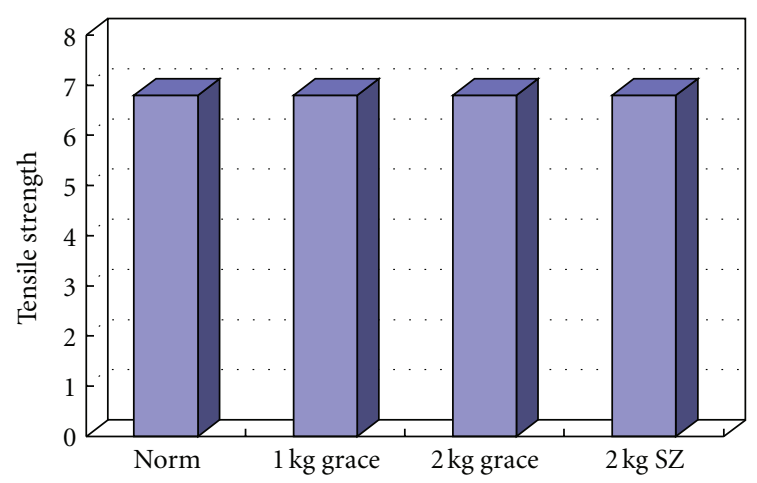

Figure 13: Tensile strength comparison.

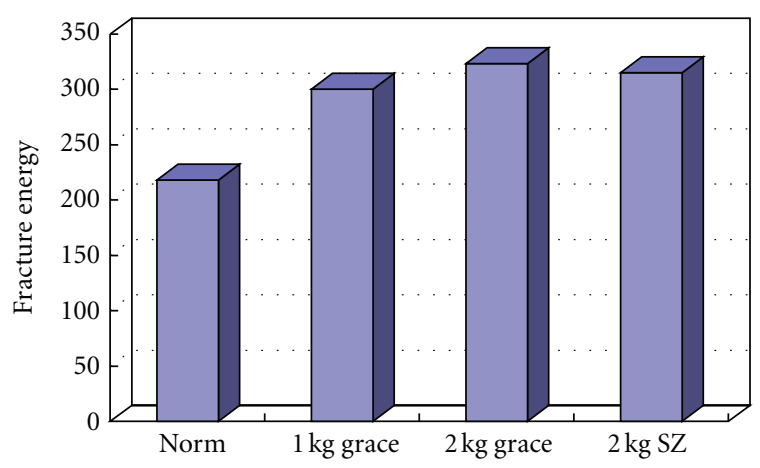

FIGURE 14: Cracking energy comparison.

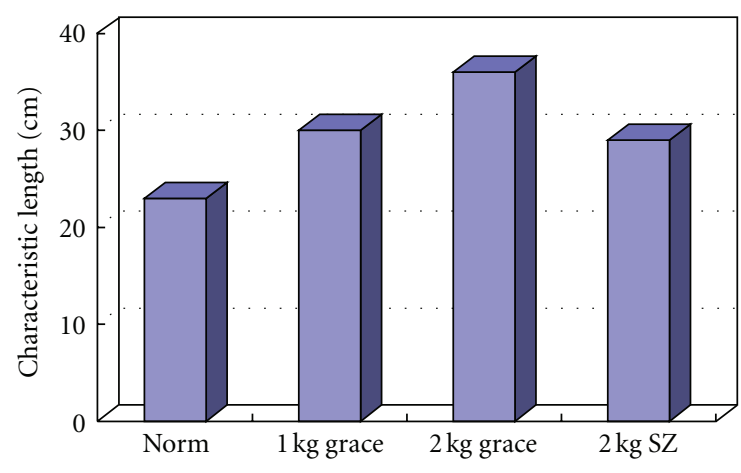

FIGURE 15: Characteristic length comparison.

$<$ concrete with $2 \mathrm{~kg}$ short fiber $<$ concrete with $2 \mathrm{~kg}$ long fiber.

\section{Conclusions}

(1) C120 ultra-high-performance concrete's fracture toughness can be enhanced by adding fiber, and as fiber content increases, cracking energy mounts too. When fiber content stays the same, fiber type makes difference too.

(2) Characteristic length of brittleness notates the brittleness of concrete. The smaller the number is, the more brittle the concrete is. As fiber content increases, concrete's toughness enhances too.

\section{References}

[1] N.-Q. Feng, High-Performance Concrete Structure, Mechanical Industry Publishing House, 2004.

[2] Y.-L. Sun, N. Xu et al., "Research on polypropylene fiber's impact on concrete toughness," Engineering Technology, no. 32: 53, 2007. 

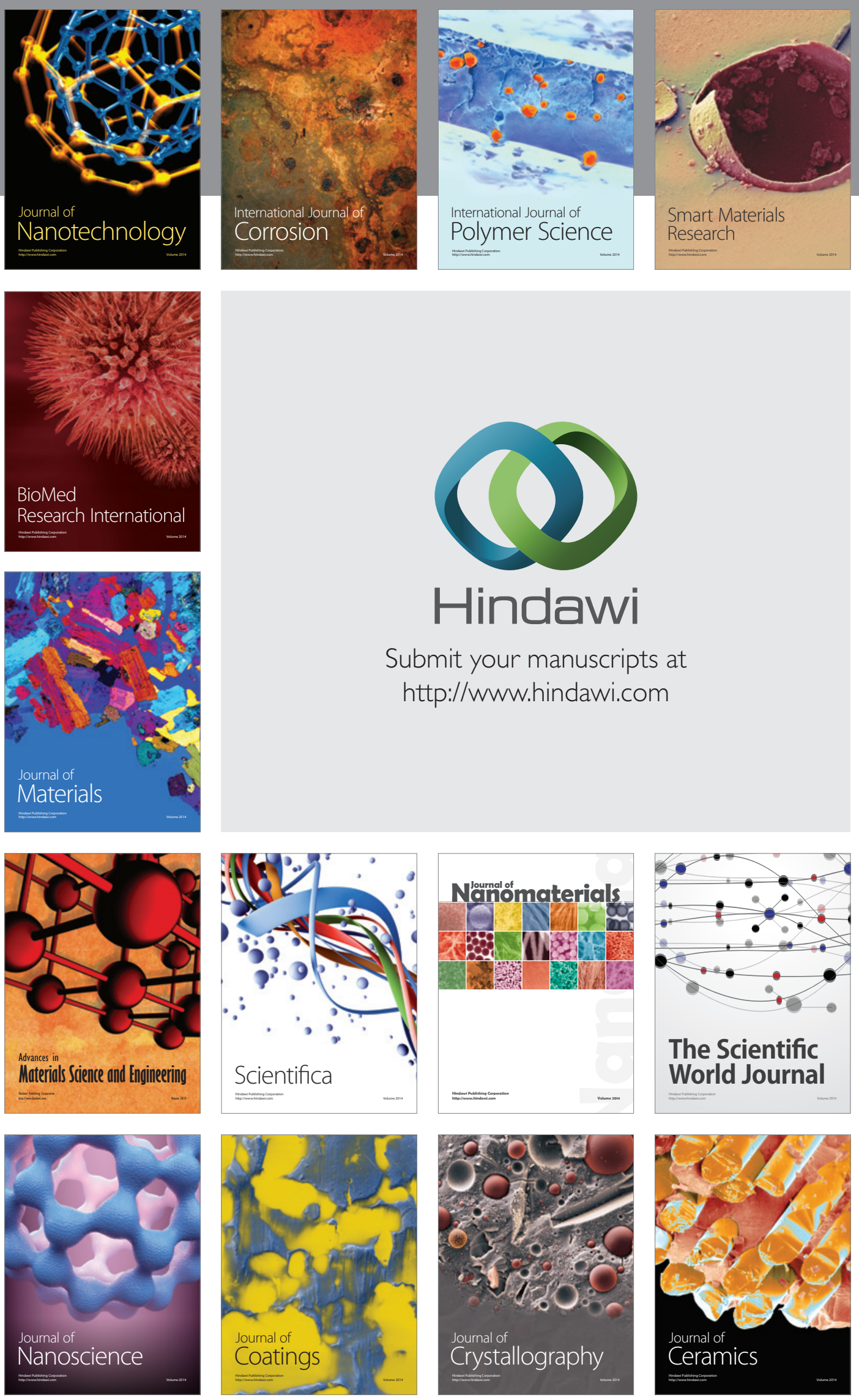

The Scientific World Journal

Submit your manuscripts at

http://www.hindawi.com

\section{World Journal}

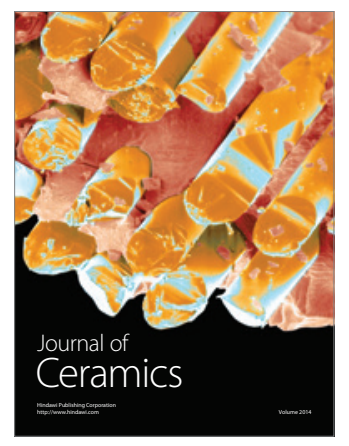

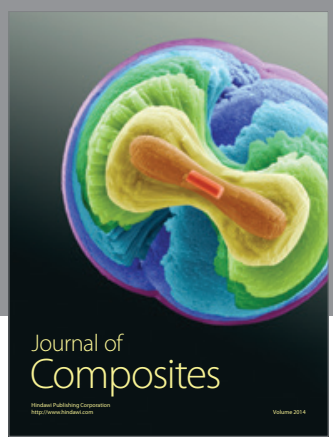
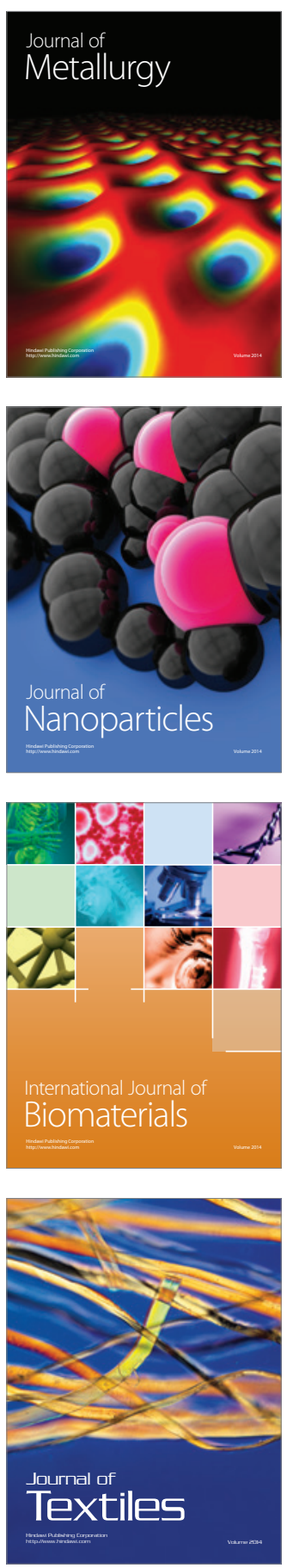